\title{
Trauma Center Imaging Problems: Proposed Solution With Picture Archiving Communication Systems
}

\author{
Robert E. Gold, Helen T. Winer-Muram, Scott L. Baum, Dale E. Hansen, S. Greg Jennings, and \\ Timothy C. Fabian
}

\begin{abstract}
Radiologic support for trauma center activities presents special problems that are discussed. This article proposed the use of a picture archiving communication system (PACS) as a potential solution. A sample PACS for this purpose is described to illustrate this approach.
\end{abstract}

Copyright $\odot 1991$ by W.B. Saunders Company

KEY WORDS: PACS, digital imaging, trauma center, trauma.

$\mathbf{M}$ ANY OF THE problems in medical care related to imaging are severely exacerbated in the setting of a level 1 trauma center. This accentuation of problems is the result of the high speed in which health care is delivered in a trauma setting and the concentrated focus of multiple surgical subspecialists and radiologists on the same small number of medical images.

The nature and extent of the imaging problems in a level 1 trauma center have been defined and are presented in this communication along with the hypothesis that a PACS system can remedy most of the known difficulties.

Although trauma has been recognized as the third leading cause of death overall in the United States, it is the leading cause of death in persons less than 40 years of age. There are approximately 150,000 trauma deaths per year. About 50,000 of these occur on the highway, with victims suffering severe multiple system injury. For every death, 2 to 3 patients suffer major long-term disability. An important estimate states that 1 in 4 of these deaths could be prevented. ${ }^{1}$ With this unfortunate fact in mind, trauma systems have been developed across the country over the past decade.

A trauma system consists of (1) a sophisticated prehospital system with paramedics initiating resuscitation and rapid transport; (2) online communication networks linking prehospital personnel with physicians in trauma centers who provide medical control; and (3) the trauma center, which acts as the hub of the system by coordinating the prehospital efforts and providing definitive trauma care. The trauma center concept has been developed and defined by the American College of Surgeons, which established three tiers: Levels I, II, and III, with the most severely injured patients being triaged to Level I institutions. Since death and major disability occur within a short time following injury, in those patients, diagnosis and therapy must be both simultaneous and expeditious. This is accomplished by having surgeonstrauma surgeons, neurosurgeons, orthopedic surgeons and anesthesiologists-and operating room teams present in the hospital at all times.

\section{THE PRESLEY LEVEL I TRAUMA CENTER}

\section{Level I Trauma Center Description}

The Presley Memorial Trauma Center (Memphis, TN) is a facility attached to the Regional Medical Center at Memphis and is comprised of the following components: two Shock/Trauma suites capable of handling four simultaneous patients; one trauma assessment section designed to handle less severely injured patients; four operating room suites; three recovery room beds; eighteen Trauma Intensive Care Unit beds; one fracture room; and one suture room.

Housed in the same facility is a large and busy emergency room, a clinical chemistry laboratory, administrative offices, on-call rooms, conference rooms and the three-room Trauma Radiology Department located $100 \mathrm{ft}$ from the shock/trauma section. The Trauma Radiology Department has its own reporting room, waiting areas, film processing area, and technical office. Two computed tomography (CT) scanners serve the entire hospital but are located immediately adjacent to the Trauma Center so that patients can be moved into CT without leaving the

From the University of Tennessee, Memphis, The Regional Medical Center at Memphis, Departments of Radiology and Surgery, The Regional Medical Center at Memphis, University of Tennessee, Memphis.

Address reprint requests to Robert $E$. Gold, $M D, R m 150$

Chandler, 800 Madison Ave, Memphis, TN 38163.

Copyright $\odot 1991$ by W.B. Saunders Company

0897-1889/91/0402-0003\$03.00/0 
Trauma Center. Unfortunately, the CT section is not physically adjacent to the remainder of the Trauma Radiology Department.

The Trauma Radiology department performs approximately 55,000 radiographic examinations per year. Of these, 15,000 originate from the Trauma Center. In addition, approximately 6,000 CT studies are performed each year, 2300 of them for the Trauma Center.

The installed Trauma Center PACS manages only the examination workload generated from the Trauma Center (not the emergency room).

\section{TRAUMA CENTER IMAGING: MEDICAL CARE PROBLEMS}

\section{Quality of Images}

The Trauma Center admits 3400 patients per year. An additional 1500 patients are seen in the Trauma Center, treated and discharged. Thirtyfive percent of radiographic studies on these 4900 patients are obtained with portable radiographic equipment. Accurate figures are not available for inpatients alone, but $50 \%$ portable radiography would not be unreasonable. The quality of radiography is extremely variable and somewhat unpredictable because of the very nature of portable radiographic equipment, the adverse conditions of the environment in Shock/ Trauma and the extreme conditions of many of the patients.

\section{Availability of Images to Surgical Services and Radiologists}

Due to the urgency involved with many patients upon arrival in the trauma center, surgeons attending these patients in the Shock/ Trauma section need to, and properly should, view films as soon as possible after the examination has been completed. These films are obtained with portable equipment, returned to the Trauma Radiology Department, processed, then transported back to the Shock/Trauma room. Commonly, the surgeons or their appointees, come to the Trauma Radiology Department and take some of the films back to shock/trauma room while other films are still being processed. Similar scenarios occur with Trauma Intensive Care Unit (TICU) patients, and with CT scans and vascular/interventional procedures on both Shock/Trauma and TICU patients. The problem of constantly moving films is less serious in the CT section because original quality images are easily and quickly reprinted. The problem is also of lesser importance for the vascular/ interventional service because of the direct radiologist to surgeon contact that occurs on that service and the lower volume of procedures.

From the Shock/Trauma section, patients may go directly to the operating room and from there to TICU and the films accompany them. The patient may be immediately attended by, or assigned to the care of, the orthopedic or neurosurgical services, in which case these surgical subspecialists are handed some or all of the patient's imaging records.

Control and filing of medical images is not a priority with any of the surgical subspecialists and images from these severely injured patients may be left behind in any area visited by either the patient or the surgeon during the acute phase of the patient's care. These areas may include other buildings, offices, or hospitals where a surgeon has gone to consult with other members of his subspecialty.

Less severely injured patients are initially evaluated in the Trauma Assessment area of the Trauma Center. These patients are generally transported to the Trauma Radiology department for imaging and, if normal, their images are virtually always viewed and left within the radiology department. If abnormal, they are frequently removed to be reviewed beside the patient or for consultation with other consultants.

Images left by one surgical service in any location other than in the Trauma Radiology department delays radiologic interpretation and potentially compromises patient care. If the images are not in the Trauma Radiology department and not beside the patient, they are also unavailable to the other surgical services with further potential for compromise in patient care.

\section{Diagnostic Radiology Results Reporting}

At the onset of the program to implement a PACS system in the Trauma Center in the spring of 1988, an assessment of the timeliness of radiology department reporting gave startling results. Two studies were performed.

The first examined the length of time from 
order written on the chart to the time a report was posted on the chart. This study excluded the Trauma Center since there is rarely time for audit personnel to chart the time interval between requests for studies in the Trauma Center and the time at which it is performed. Nevertheless, the same dictation system, transcription system and report posting system applied and extrapolation of these results to the Trauma Center was assumed valid. This study showed an average time from examination request to results posted of over 4 days. Virtually the entire delay was attributed to delayed transcription of dictated reports and delayed posting of signed reports. As a result of this study, additional transcriptionists and dedicated radiology posting clerks were hired. A repeat audit in the fall of 1989 showed $100 \%$ of reports were posted on the patient chart within 24 hours.

The second study determined the number of examinations performed in each section of the Trauma Center that were unreported. This study was limited to the Trauma Center. Figures were obtained for April and May of 1988. The study was performed using data from the Radiology Information System and was performed during the first week of June, 1988. The results are shown in Table 1 and include only conventional radiographic studies. Overall, 18\% of all examinations in the study period were unreported. The unreported studies were slightly higher in April than in May, indicating that once unreported, these studies did not reappear in a fashion leading to late reporting. It is assumed that these unreported films represented examinations lost or misfiled when removed from the Trauma Radiology Department.

\begin{tabular}{lccccc}
\multicolumn{2}{c}{ Table 1. Unreported Trauma Center Imaging Studies } \\
\hline & Shock & Trauma & Trauma & \\
April, 1988 & Trauma & ICU & OR & Totals \\
\cline { 2 - 4 } No. of procedures & 485 & 589 & 138 & 1212 \\
No. unreported & 132 & 34 & 67 & 233 \\
Percent unreported & $28 \%$ & $6 \%$ & $49 \%$ & $19 \%$ \\
& & & & \\
& Shock & Trauma & Trauman & \\
May, 1988 & Trauma & ICU & OR & \\
No. of procedures & 492 & 645 & 109 & 1246 \\
No. unreported & 122 & 39 & 41 & 202 \\
Percent unreported & $25 \%$ & $7 \%$ & $38 \%$ & $16 \%$ \\
\hline
\end{tabular}

NOTE. Conventional films only; does not include CT.
Unreported examinations not only expose a patient to lack of diagnostic radiology input, but expose the hospital and all physicians caring for a patient to medico-legal problems. In addition, the financial loss to the hospital and the radiology department is considerable.

As a result of the study on unreported examinations, clerks were assigned to the Trauma Radiology Department 24 hours a day, 7 days a week. They are responsible for rapid organization of examinations for reporting and frequently visit all areas of the Trauma Center picking up and reorganizing radiographic images. Re-examination of unreported studies in the fall of 1989 in the same manner as described above showed an overall unreported film rate of $6 \%$.

Despite the marked improvements in speeding reports to the patient chart and reporting of Trauma Center radiographs, the Departments of Radiology and Surgery agree that more rapid and reliable communication is needed for optimal care in this setting. Optimal care should mean that a typed report is appended to an image the moment it is dictated as well as the earliest possible verbal communication of findings between radiologist and surgeon. One reasonable alternative is once-daily review, together, of current images of patients studied in the Trauma Center. Storage of plain film, CTs and vascular/interventional procedures in a single, easily displayed fashion would greatly enhance the value of regular trauma conferences or "working rounds."

TRAUMA CENTER IMAGING: FINANCIAL PROBLEMS

\section{Staffing Expenses}

Due to the often frenetic pace of Trauma Center activities and the uncertainty of the location of many radiographic examinations at any particular time, significant amounts of time is spent collecting and collating radiographic examinations by technologists, clerks and radiologists. This concentration of people, however, has resulted in marked improvement in measurable performance in the Trauma Center as described above. 


\section{Repeated Films Due to Poor Radiographic Technique}

A high percentage of examinations obtained with portable equipment require repeat studies simply because of the nature of portable radiography. As noted earlier, this is exacerbated in the Shock/Trauma section because of the difficulty of the circumstances surrounding an emergent resuscitation. In addition to the pure cost of repeating the films, the cost of the delays in diagnosis cannot be estimated.

\section{Repeated Examinations Due to Lost Films}

When radiographic images are unavailable because one surgical team has them and a radiologist has not reported them, other surgeons caring for a patient order repeat examinations. By experience, they have found it faster to repeat radiographic studies than to try to locate missing films.

\section{Additional Studies Compensate for Inadequate or Equivocal Studies}

It has become common practice to order additional imaging studies such as CT rather than repeating some technically poor radiographic studies. This is often the case with facial views and especially true for cervical spine studies where knowledge of a normal cervical spine is critical information in the ongoing management of the severely traumatized patient. With technically satisfactory studies, or the option of image processing, some of these redundant $\mathrm{CT}$ and radiographic examinations may be eliminated.

\section{Risk of Billing for Unreported Imaging Studies and Lost Revenues}

The Trauma Center bills on the basis of "examination complete." The radiologists bill for professional services on the basis of "report dictated." Insurance carriers audit on the basis of "report on medical record" as well as other criteria. In the event of an insurance carrier audit, films unreported because they were removed from Trauma Radiology and mishandled represent a significant financial risk to the hospital in terms of revenue that must be returned. The radiologists, of course, have never billed for those studies at all.

\section{TRAUMA CENTER IMAGING: LEGAL AND MEDICO-LEGAL PROBLEMS}

By its very nature, the Trauma Center is an area with a high risk of legal entanglement, with a significant chance of legal action related to events surrounding the trauma as well as a risk of medico-legal action. Unavailability of radiographs and reports to assist the trauma team in treatment or to support a patient's action against a third party responsible for an injury must increase the hospital's and physicians' legal exposure.

\section{SUMMARY}

\section{Trauma Center Imaging: The Problems}

The major medical care problems outlined above can be summarized as a problem in control of the medical image. For optimal care, medical images should be available to the radiologist and to all other physicians caring for the patient, simultaneously and at all times.

Inconsistent image quality is a less important problem than no image at all. In a trauma center where a significant proportion of films are obtained with portable radiography, anything that improves consistency would be valuable.

Rapid and reliable reporting is virtually synonymous with the appropriate practice of radiology. It is also impossible if the images to be reported are not available. Good direct radiologist-to-clinician communication is also difficult if images of a single patient are scattered amongst three physically separated imaging areas: the Trauma Radiology Department, the CT scanner and the Vascular/Interventional procedures rooms.

The major financial problems and risks once again relate to lack of control of the medical image-unbilled studies or studies billed but not reported. The clerical staff needed to organize and retrieve radiographic studies represents another very large financial consideration. Repeated films, repeated examinations and additional studies certainly add up to an important financial drain over a period of time. 
Legal and medico-legal risks are clearly exacerbated by lack of control of the medical images.

\section{PACS AS A PROPOSED SOLUTION TO TRAUMA CENTER PROBLEMS}

In an attempt to resolve the many imaging problems in the Presley Trauma Center, a PACS system was specified, acquired, and installed in December, 1989 (Table 2). (Since submission of this article, a relatively low-cost personal computer-the Siemens (Iselin, NJ) "LightBox"-has been added to the PACS system.) In addition, a technical supervisor and three clerks were hired to manage the hardware and software.

\section{Computed Radiography and Digital Data}

Since lack of control of the trauma center images was identified as a major contributor to the problems, a computed radiography (CR) system was considered to be the single most

Table 2. Components of Installed Trauma System PACS

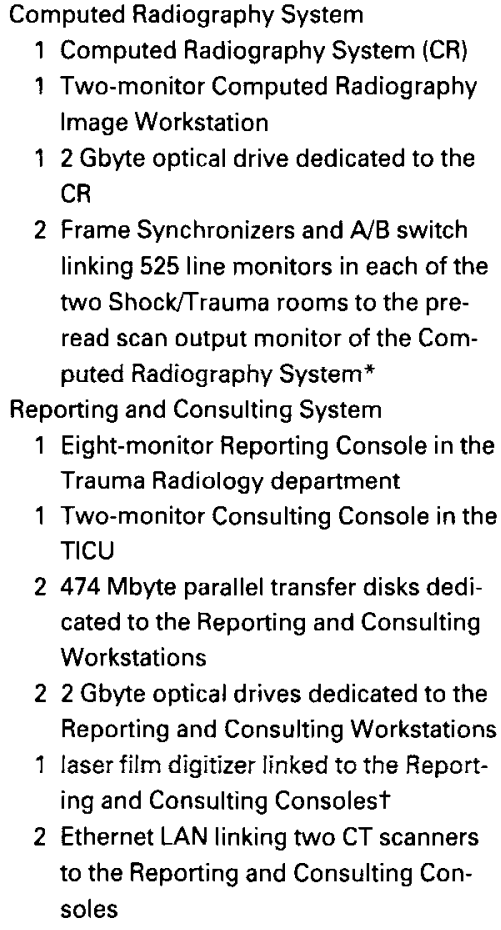

2474 Mbyte parallel transfer disks dedicated to the Reporting and Consulting Workstations

22 Gbyte optical drives dedicated to the Reporting and Consulting Workstations

1 laser film digitizer linked to the Reporting and Consulting Consolest

2 Ethernet LAN linking two CT scanners to the Reporting and Consulting Consoles

NOTE. All components from Siemens Corporation (Iselin, NJ) unless otherwise specified.

tDupont Corporation, Wilmington, DE.

*Pro-Video, Memphis, TN important component of the PACS solution. Furthermore, an essential characteristic of the selected CR system was that it retained the original data in digital form, permitting reprinting of original quality images and transmission of images to display workstations. These features should allow the system to meet the stated goal that medical images should be available to the radiologist and to all other physicians caring for the patient, simultaneously and at all times.

Current CR systems process images more slowly than conventional radiographic systems. In a trauma center, rapid access to images is critical, particularly in the Shock/Trauma section where incoming patients are being resuscitated. In order to compensate for the longer processing time of the installed CR system, it was fitted with digital frame grabbers attached to the "preread" video output. The frame grabbers are connected to low-resolution monitors (525 line) in the Shock/Trauma rooms allowing transmission of selected studies and resulting in faster delivery of images than could be accomplished with a conventional system. The lowresolution monitors are considered adequate for delineation of major, life threatening abnormalities until the printed images arrive.

In practice, the Trauma Center is only one component of the hospital, and so film-based archiving will continue to operate in parallel with the PACS system. All patients in the Trauma Center will have films printed and filed in the same manner as in the past. In addition, the images will simultaneously be archived to optical media.

\section{Multi-Modality Display Workstations}

The ultimate purpose of preventing loss of images and of making images available at all times to both surgeons and radiologists is to promote communication amongst physicians caring for the patient. Communication, in the Presley Trauma Center is further hindered by the fact that the CT section, the Trauma Radiology Department, and the Special Procedures suites are all remote from one another.

In order to resolve the communications problems, a mechanism was needed to bring all images on the trauma patient to one location in a fashion that could permit consultation, confer 
encing, and interpretation. An eight-monitor Siemens DRC-80 was installed in the Trauma Radiology Department, and a two-monitor Siemens DRC-20 console was installed in the TICU. These and any future display workstations represent the reporting and consulting components of the Trauma Center PACS. All CT studies performed on Trauma Center patients will be printed, archived, and reported as before. In addition, these CT studies will be immediately transferred to the reporting and consulting stations so that the studies are available to radiologists and surgeons in the Trauma Center.

Until low-cost consulting workstations are available, image viewing in the Trauma Operating Rooms will continue to be film based. It is expected that appropriate workstations will become available for this application within the next 12 months.

\section{Laser Digitizer}

$\mathrm{CT}$ and $\mathrm{CR}$ are primary digital imaging modalities that are compatible with the installed reporting and consulting system. In order to make special procedures studies (noncompatible digital system) and outside radiographs available on the display workstations along with the other images, a high-resolution analog to digital converter was required and, consequently, a Dupont Laser Digitizer (Dupont, Wilmington, DE) was installed as part of the PACS system. The radiologists in the trauma center will select pertinent films from outside studies and the Angiography/Interventional Service will select a limited number of special procedures images for digitization on trauma patients.

The laser digitizer will serve the additional utility of converting analog films obtained during periods of time when the CR system is disabled for any reason.

\section{Image Storage}

The Trauma PACS system is equipped with 958 Mbytes of high-speed parallel transfer magnetic disk space suitable for rapid loading of patient's images onto the display workstations. This will permit temporary storage and rapid retrieval of the equivalent of about $100 \mathrm{CR}$ images. (Since submission of this article, a much expanded magnetic disk storage capability is being considered for the system. This additional high-speed storage could impact on the personnel needed to organize the system.) Two optical disk drives are also installed, each providing 2 Gbytes of data storage. However, because of the slow transfer rate from optical drives to the workstation, this storage capacity is considered useful only for archiving or for retrieving images in preparation for planned conferences.

\section{Personnel}

The display workstations and their associated high-speed magnetic disks are intended to perform a variety of clinical functions:

(1) accept CT images transferred from the CT department; (2) accept selected digitized images from outside sources; (3) accept selected digitized images from special procedures; (4) store and organize current day and selected previous images on all TICU patients; (5) store and organize all images on patients with minor trauma until reporting is completed; and (6) bring together a variety of images from multiple patients for conference purposes.

Current software available for display workstations provides minimal intelligence in organizing the limited high-speed disk space for clinical activities. It is expected that considerable time and effort will be expended by the electronic equivalent of file room staff in order to meet the demands expected of the display workstations. Consequently, a "PACS Supervisor" and three additional clerical staff were hired in order to assist technologists, surgeons, and radiologists in the use of the PACS system and to organize and maintain the magnetic and optical disk drives so that the system can meet expectations. After extensive training, the supervisor and the clerks will staff the PACS system two shifts per day during the week and one shift per day on the weekends.

\section{Integration of the PACS System with the Rest of the Hospital}

When a patient leaves the Trauma Centereither transferred to the general hospital population or discharged home-the film records (hard copies) will be merged with all other 
hospital film records. Lost hard copies of the images will be reprinted from the Trauma Center optical archives only when a need for those records is recognized.

\section{RESOLVING MEDICAL CARE PROBLEMS}

Acquisition of images in digital format with immediate storage on magnetic and optical media provides the reliable control of the images needed to resolve most of the medical care problems already defined. Images misplaced or removed from the Trauma Radiology Department can be easily reproduced. Soft copies of the image will always be available to radiologists and surgeons at both viewing components of the reporting and consulting system. Hard copies can be reprinted as required. All images on all patients will be maintained in an easily accessible digital format as long as the patient remains within the Trauma Center.

There still remains the chance that a small number of images will be lost during periods of time when the CR system is not operating and conventional films must be collected and digitized. This number of lost images should only be a small fraction of the current volume.

The Trauma System PACS has no integrated dictation system currently. Reports will be dictated on conventional dictation equipment and transcribed and distributed in the same fashion as is currently used. Until direct voice to text dictation systems are available and integrated with our PACS system, communication between radiologist and trauma surgeon will be less than optimum. In the interim, the eight-monitor Diagnostic Reporting Console in the Trauma Radiology department will serve as the site of daily working conferences for review of all imaging studies on trauma patients. With all conventional images, CTs, outside films, and special procedures studies available for daily conferencing, it is believed that the medical care problems, previously described, can be remedied.

\section{Resolving the Financial and Legal/Medico-Legal Problems}

Those financial, legal, and medico-legal problems that are related to control of the medical images should be resolved in the same fashion and to the same extent as are the medical care issues described above.

As expected from the experience of others and detailed earlier, staffing expenses at the University of Tennessee Regional Medical Center have increased with installation of the Trauma Center PACS system. ${ }^{2}$ It remains to be seen whether any decreases in staffing expenses will be effected in the future.

Based on the known benefits of CR and the additional image processing capabilities of both the CR and the Reporting and Consulting Systems, repeat film studies should be cut to a minimum and the additional studies performed due to inadequate technique should decrease..$^{3-5}$

\section{Missing Components of the Trauma Center PACS}

A number of important components are not currently a part of the University of Tennessee Regional Medical Center's Trauma Center PACS. Some but not all of these have already been noted above and some but not all are planned for our system in the future: (1) lowcost image workstations in the trauma operating rooms; (2) integration of the Radiology Information System (RIS) with the Trauma Center PACS; (3) voice-to-text dictation with integration into both the RIS and the PACS; and (4) integration of third-party Digital Subtraction Angiography with the Trauma Center PACS.

\section{EVALUATION OF THE TRAUMA CENTER PACS SYSTEM}

A number of criteria must be met to determine that PACS is effective in resolving the imaging problems in a trauma center: (1) CR must be determined to be of appropriate quality to replace conventional imaging; (2) unreported imaging studies should be dramatically reduced; (3) repeat examinations and studies of marginal quality should be reduced; (3) unnecessary additional imaging studies might be measurably reduced; (4) users of the system should perceive that their patients' examinations are always available to them and are rapidly and easily retrievable. How well the Trauma Center PACS System meets these criteria will be the subject of future communications. 


\section{REFERENCES}

1. Hospital and prehospital resources for optimal care of the injured patient. Bulletin of the American College of Surgeons. 71:4-12, 1986

2. Saarinen AO, Goodsitt MM, Loop JW: The logistics of installing PACS in an existing medical center. Proceedings of the SPIE 1093:159-170, 1989

3. Taira RK, Cho PS, Huang HK, et al: Performance evaluation of a clinical PACS module. Proceedings of SPIE 1093:406-415, 1989

4. Merritt CB, Tutton RH, Bell KA, et al: Clinical application of digital radiography: Computed radiographic imaging. Radiographics 5:327-414, 1985

5. Schaefer CM, Greene RE, Oestmann JE, et al: Improved control of image optical density with low-dose digital and conventional radiography in bedside imaging. Radiology 173:713-716, 1989 